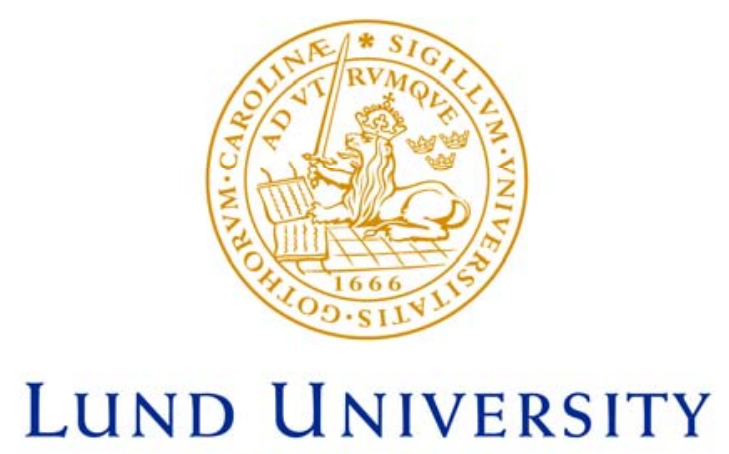

Faculty of Medicine

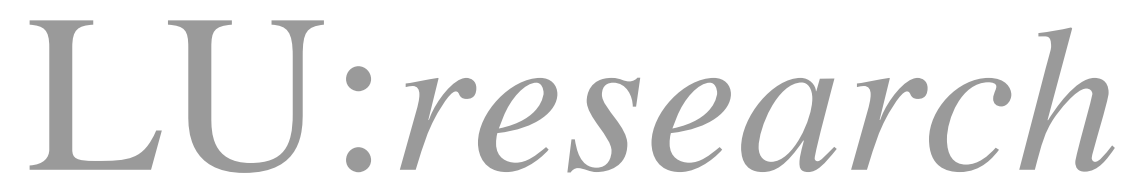

Institutional Repository of Lund University

This is an author produced version of a paper published in Acta Paediatrica. This paper has been peer-reviewed but does not include the final publisher proof-corrections or journal pagination.

Citation for the published paper:

Stenram, Unne and Cramnert, Catarina and Axfors-Olsson, Helene.

"Oralfacialdigital-like syndrome with respiratory tract

symptoms from birth and ultrastructural

centriole/basal body disarray"

Acta Paediatr, 2007, Vol: 96, Issue: 7, pp. 1101-1104.

http://dx.doi.org/10.1111/j.1651-2227.2007.00326.x

Access to the published version may

require journal subscription.

Published with permission from: Blackwell 


\section{Oralfacialdigital-like syndrome with respiratory tract symptoms from birth and ultrastructural centriole/basal body disarray}

Unne Stenram $^{1}$, Catarina Cramnert ${ }^{2} \&$ Helene Axfors-Olsson ${ }^{3}$

${ }^{1}$ Department of Pathology, Lund University, Lund, Sweden, ${ }^{2}$ Department of Pathology and Cytology, University Hospital, Lund, Sweden, ${ }^{3}$ Department of Paediatrics, Central Hospital, Växjö, Sweden.

Correspondence: Unne Stenram, Department of Pathology, Lund University, University Hospital, SE-22185 Lund, Sweden. Tel :+46 46 173407. Fax: +46 46 143307. E-mail: unne.stenram@med.lu.se.

Running head: Centriole/basal body disarray 


\section{Abstract}

A girl with polydactyly has had respiratory tract problems, including atelectasis, since birth . She has a high arched palate, a tongue hamartoma and dysmorphic face. Electron microscopy of nasal and bronchial brush biopsies repeatedly revealed centriole/basal body disarray and extreme sparseness of cilia. At the age of 2 years and 11 months she displays retardation of both motor and mental skills.

Conclusion: The manifestations tally with a ciliopathy, partly with the Bardet-Biedl syndrome but especially with the oralfacialdigital syndrome; however, with the addition of persistent respiratory tract problems. As these two syndromes are considered to be due to mutations affecting the centriole/basal body apparatus, the ultrastructural demonstration of disarray of these structures, never before demonstrated in such a patient, is of fundamental interest.

Key Words: Ciliopathy, oralfacialdigital syndrome, Bardet-Biedl syndrome, respiratory tract cilia, ultrastructure, centriole/basal body 


\section{Introduction}

There are several ciliopathies [1], two of them with polydactyly, oral malformations, dysmorphic face and neurological symptoms, namely Bardet-Biedl syndrome (BBS) [2] and the oralfacialdigital syndrome (OFDS) [3]. The mutations in these diseases affect the centriole/basal body complex of the cilia, in several tissues and organs [1, 4]. It is therefore remarkable that respiratory tract symptoms due to failing cilia are not reported in patients. Asthma is described in BBS [2] but not listed among the diagnostic criteria.

We have encountered a girl with polydactyly, a high arched palate, tongue hamartoma, dysmorphic face and atelectasis with suspicion of pneumonia from birth. At the age of 2 years and 11 months, she displays retardation of motor and mental skills. Repeated ultrastructural examination of nasal and bronchial brush biopsies revealed disarray of the centriole/basal body complex and very few cilia in the columnar epithelial cells. Though more manifestations might eventually appear, it was considered of interest to present the case, as such ultrastructural abnormalities of respiratory tract cilia have not, to our knowledge, previously been reported in this type of syndrome. 


\section{Case report}

As a result of bad progress of labour, acute Caesarean section was performed in week $42+3$. Apgar score was 3 - 7 - 10. Meconium-coloured amniotic fluid was withdrawn from the trachea. The birth weight was $3245 \mathrm{~g}$. The girl presented with postaxial polydactyly. She has a high arched palate and a tongue hamartoma. She does not have situs inversus. She has no siblings. The parents are not consanguineous. The girl has a dysmorphic face (Figure 1).

The girl had respiratory problems from the first day, needed oxygen during the first month and was supported by low continuous positive airway pressure (CPAP). Inspiratory sounds suggested laryngomalacia, especially during the first weeks. Pulmonary X-ray was normal on day 1 but on day 6 there were pulmonary parenchymal changes with suspicion of pneumonia, and atelectasis in several lobes in the second week, which persisted for many weeks. She always presents plenty of mucus and is regularly given breathing exercise, and inhalation with bronchodilating and mucolytic medicine. She often receives antibiotics too. At 10 months she had slight-moderate bronchiectasis on computer tomography. The right upper lobe bronchus was found to start from the main bronchus, and a segment bronchus in the same lung also had an anomalous origin. At 2 years and 9 months there was still atelectasis in the middle lobe and mediobasally in the left lung, and she has had several upper airway infections and bronchopneumonia in the last two months. She is now in good condition.

She vomited a lot during the first year and had a gastrostomy from 11 to 33 months because of poor weight increase. She has repeatedly had fluid in the middle ears and has twice been subjected to paracentesis, which has yielded a clear viscous secretion. There were a few spots of mild, unspecified atrophy in her retinae at 4 months. 
The girl has hypotonic muscles and delayed motor progress and cannot walk at 2 years and 11 months. She speaks only a few words but is active and curious about things. Magnetic resonance tomography shows a slightly enlarged fourth ventricle. EEG was normal at the age of 5 days. Renal, hepatic and splenic ultrasound was normal at 2 years and 10 months of age. Gene test for Prader-Willis syndrome was negative. Subtelomere fluorescence in situ hybridization (FISH) test was negative. There is no immunological deficiency. Nasal nitric oxide (NO) has not been examined because of her young age. Nasal brush biopsies for electron microscopy were taken three times, at the ages of 2 months, 10 months and 21/2 years. At 10 months, a bronchial brush biopsy was also taken. They were fixed in $2 \%$ glutaraldehyde with $2 \mathrm{mM} \mathrm{MgSO}_{4}$ in $0.1 \mathrm{M}$ sodium cacodylate buffer with $0.1 \mathrm{M}$ sucrose at $\mathrm{pH} 7.2$ and postfixed in $1 \% \mathrm{OsO}_{4}$. The sections were contrasted with uranyl acetate-lead citrate. The ultrastructural findings were the same on all occasions. The columnar epithelial cells showed good cell vitality with normal mitochondria. They were predominantly covered by microvilli (Figure 2 in Supplementary Material online). The few cilia had a normal orientation of the central microtubuli and a normal substructure with a mean of 7.7 outer and 3.4 inner dynein arms. There were a few compound cilia. Cilia were also found in the cytoplasm (Figure 3 in Supplementary Material online) and occasionally together with microvilli in intracytoplasmatic cysts (Figure 4 in Supplementary Material online). Basal bodies were found in the middle part of the cells and close to vesicles (Figure 2 in Supplementary Material online). A centriole with surrounding procentrioles was encountered (Figure 4 in Supplementary Material online). There were occasional striated filaments and granular complexes of the types considered to be centriolar precursor material $[5,6,7]$. To summarise, there was a centriole/basal body disarray and sparseness of cilia. 


\section{Discussion}

Polydactyly, oral malformation, a dysmorphic face (Figure 1) and retarded motor and mental development point to a syndrome, primarily BBS and OFDS. Diagnosis of BBS requires four major or three major and two minor features [2]. Our patient displayed two major (polydactyly, mental retardation) and three minor (high arched palate, developmental delay, speech delay) features of BBS and thus does not fulfil the criteria of this syndrome. Because of her young age, more manifestations may, however, eventually appear.

OFDS may be more appropriate, as in addition she displays respiratory tract malformations. Of the eight main features of OFDS [3] she displays four or five (oral, facial, skeletal, neurological and possibly laryngotracheal; laryngomalacia, doubtful in this case, and abnormal origin of the bronchi, however, do not seem to have been described in OFDS, though laryngomalacia has been described in other syndromes [8]). Respiratory tract infections, atelectasis and bronchiectasis are not described as manifestations of BBS or OFDS. Her ear problems are probably also due to cilial defects. This girl might therefore present a new variant of OFDS. BBS cannot be excluded, as two of its major features, blindness and obesity, do not become manifest until puberty [2]. At least 12 BBS genes have been described [9], and there are at least eight types of OFDS [3]. It is therefore not surprising that new constellations of manifestations may appear. Possibly these syndromes will be renamed after the mutations involved.

BBS, OFDS, Alström syndrome and nephronophthisis (also part of Senior-Loken syndrome) are now considered to be ciliary diseases with mutations affecting the centriole/basal body complex $[1,4,9]$. The disarray of this complex in the nasal and bronchial brush biopsies (Figure 2 in Supplementary Material online) is therefore 
probably a part of the syndrome in the present case. The findings might even suggest that a mutation affecting the centriole/basal body complex is the cause of the disease. It is of interest that respiratory tract manifestations, including sinusitis and bronchopneumonia, are common in Alström syndrome [10]; however, cilia do not seem to have been examined.

There were a few cilia, with a normal substructure, but a preponderance of microvilli. Somewhat similar pictures have been described in ciliary aplasia $[5,6,11,12,13]$ considered to be a variant of primary ciliary dyskinesia - of striated roots $[5,6,12]$ and granular complexes of procentriolar nature [5] in the cytoplasm, sparsely found also in the present case, and a few basal bodies in the apical part of the cytoplasm [12], which are seen in abundance in the middle part of the cells in our patient (Figure 2 in Supplementary Material online). Several vesicles were close to the basal bodies (Figure 2 in Supplementary Material online) and might therefore have been involved, for example, in the failure of intraflagellar transport (ie transport of Golgi material to the top of the cilia thus contributing to cilial growth).

In two previous studies investigating ciliary aplasia, few cilia were observed [12, 13], as in the present case. We initially adopted the term cilia aplasia [14]. However the primary change in our patient is probably in the centriole/basal body complex and the sparseness of cilia may be secondary. We therefore propose to name it centriole/basal body disarray, as it tallies with the view of the pathogenesis of the abovementioned syndromes.

In only two cases of ciliary aplasia, were symptoms other than from the respiratory tract described; that is, blindness and deafness (Usher syndrome type 1) [11], and hearing impairment [12]. There are many types of cilia, and the ciliary proteins are variably expressed. Consequently there are great variations in the manifestations of ciliary 
hereditary diseases. Similarly situs inversus, which is considered to be a sign of primary ciliary dyskinesia, can occur without respiratory tract infection but with different types of malformations [14]. Situs inversus was not reported in the above patients with ciliary aplasia nor found in the present case.

In conclusion, the symptoms of this patient seem to be in accord with the OFDS, but with the addition of severe respiratory tract symptoms from birth and ultrastructurally a centriole/basal body disarray in nasal and bronchial brush biopsies; therefore this is a unique report. 


\section{Acknowledgements}

Computer help by Eric Carlemalm, Rolf Odselius and Thomas Lindén is gratefully acknowledged. We also want to thank the paediatricians Bill Hesselmar, Gothenburg, and Peter Meyer, Lund. The case was presented at a primary ciliary dyskinesia meeting in Lund Nov 24, 2006. 


\section{References}

[1] Badano J, Mitsuma N, Beales PL, Katsanis N. The ciliopathies: an emerging class of human genetic disorders. Annu Rev Genomics Hum Genet 2006; 7:125-48.

[2] Moore SJ, Green JS, Fan Y et al. Clinical and genetic epidemiology of Bardet-Biedl syndrome in Newfoundland: a 22-year prospective population-based, cohort study. Amer J Med Genet 2005; 132A: $352-60$.

[3] Matheny M, Hall B, Manalihod JM. Otolaryngologic aspects of oral-facial-digital syndrome. Int J Pediatr Otorhinolaryngol 2000; 53:39-44.

[4] Marshall WF, Nonaka A. Cilia: tuning in to the cell's antenna. Curr Biol 2006; 16:R604-14.

[5] Götz M, Stockinger L. Aplasia of respiratory tract cilia. Lancet 1983; i:1283.

[6] de Santi MM, Gardi C, Barlocco G et al. Cilia-lacking respiratory cells in ciliary aplasia. Biol Cell 1988; 64:67-70.

[7] Hagiwara H, Ogwada N, Takata K. Cell biology of normal and abnormal ciliogenesis in the ciliated epithelium. Int Rev Cytol 2004; 234:101-41.

[8] Chen J, Messner A, Chang K. Familial laryngomalacia in two siblings with syndromic features. Int J Pediatr Otorhinolaryngol 2006; 70:1651-5.

[9] Stoetzel C, Muller J, Laurier V et al. Identification of a novel BBS gene (BBS12) highlights the major role of a vertebrate-specific branch of chaperonin-related proteins in Bardet-Biedl syndrome. Am J Hum Genet 2007; 80:1-11.

[10] Marshall J, Bronson R, Collin G et al. New Alström syndrome phenotypes based on the evaluation of 182 cases. Arch Intern Med 2005; 165:675-83.

[11] Tosi GM, Pradal U, Luzi P. Usher syndrome type 1 associated with primary ciliary aplasia. Arch Ophthalmol 2003; 121:407-8.

[12] Lungarella G, Dallai R, de Santi MM. Clinical course and changes in respiratory epithelium in two sisters suffering from ciliary aplasia. In: Canciani M, Lungarella G, eds. Primary Ciliary Dyskinesia, Second joint meeting, Chiusaforte: La Chiusa, 1997:15-25.

[13] Gil YR, González MAM, Orradre JL. Ciliary hypoplasia: a rare cause of ciliary dyskinesia. Ultrastruct Pathol 2006; 30:401-2.

[14] Carlén B, Stenram U. Primary ciliary dyskinesia: a review. Ultrastruct Pathol 2005; 29:217-20.

[15] Afzelius BA. A human syndrome caused by immotile cilia. Science 1976; 193:316-8. 
Legends of figures

Figure 1 . The girl aged 2 years 11 months with dysmorphic features: bitemporal narrowing, short palpebral fissures, long philtrum, epicanthus and slightly downturned mouth. The hamartoma is just visible on the left edge of the tongue.

Figures 2-4. Electron micrographs of respiratory tract columnar cells.

Figure 2. An epithelial cell with microvilli on the surface. There are several dark basal bodies in the middle part of the cell. The inset displays seven of them in close contact with vesicles at 2.5 times higher magnification.

Figure 3. Cilia (arrows) in the cytoplasm (more or less well-defined). There are several dark structures of unknown nature, which may be abortive cilia. There seems to be a tendency towards cyst formation. Normal mitochondria are seen in the upper left corner of the photograph.

Figure 4. A well-delimited intracytoplasmatic cyst with microvilli. There is a centriole surrounded by six procentrioles. To the left is a centriole-like structure.

Figure 5. Cross-section of nasal cilium. Each of the named structures can be defective or absent as a result of mutations.

Oeri was the first to describe in 1901 what has since 1933 been known as Kartagener syndrome - following a more detailed description of the syndrome by Kartagener - (i.e sinusitis, bronchiectasis and situs inversus). Male patients in particular are often infertile. In 1975, Afzelius [15] found immotility of and ultrastructural defects in the sperm and respiratory tract cilia in these patients. Situs inversus is now known to be 
caused by immotility of the cilia in the embryonic (Hensen's) node. That makes the laterality of the body random, and $50 \%$ of the patients should have situs inversus. There are at least 13 different defects in the cilia, which are not always totally immotile. Situs inversus therefore occurs in less than $50 \%$ of cases. The disease is now called primary ciliary dyskinesia.

The ultrastructural examination is mainly made on cross-sections of the cilia. The most common defects are absence of outer and/or inner dynein arms (Figure 5). These must be distinguished from changes due to infection. Other diagnostic findings are impaired ciliary motility and decreased nasal NO levels.

Cilia of different types are present in most cells and tissues of the body. Diseases caused by mutations of the ciliary genes are called ciliopathies. Sometimes mainly one organ is affected as in autosomal dominant and autosomal recessive polycystic kidney disease. As a rule, many organs and tissues are affected with a great variation in clinical manifestations described as various syndromes, some of which are very unusual; for further details see Badano et al. [1] and references therein. Commonly these syndromes do not include respiratory tract symptoms, but situs inversus is occasionally described. The mutations can also affect the root system of the cilia, the centriole/basal body, as in the present case.

Electron micrograph by Birgitta Carlén, Department of Pathology, Lund. 


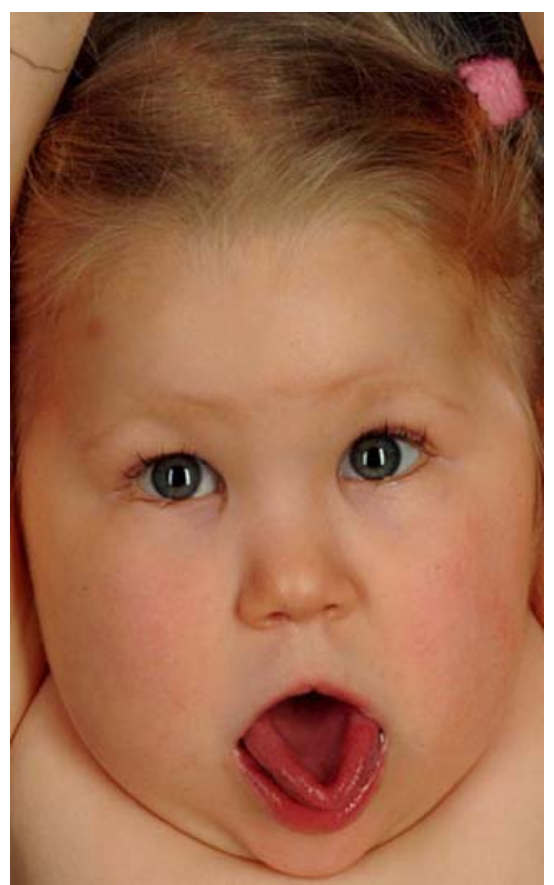

Figure 1

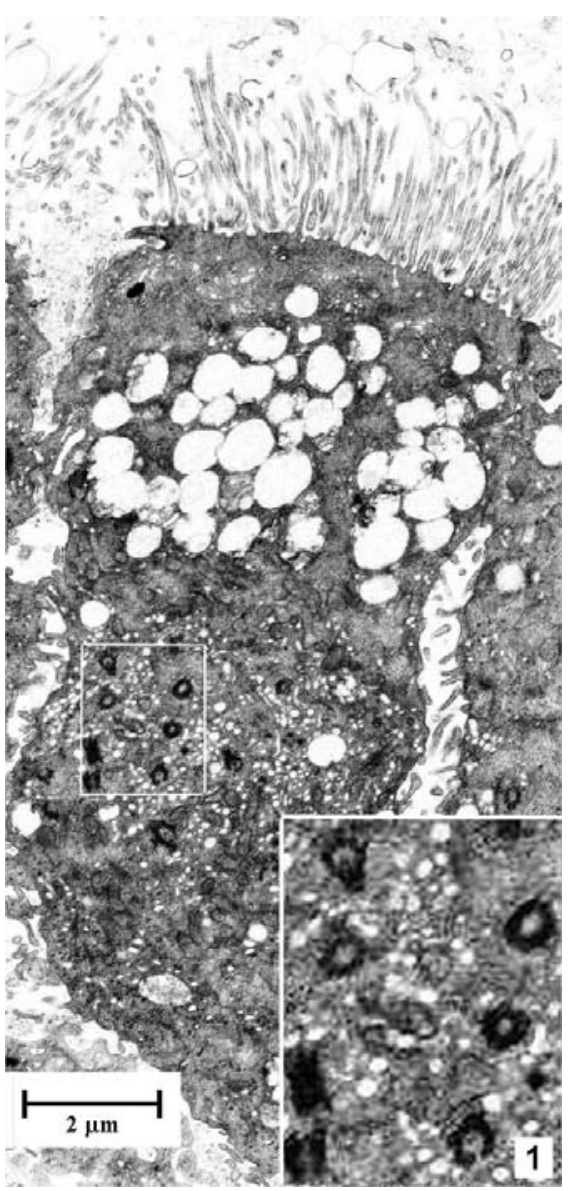

Figure 2 


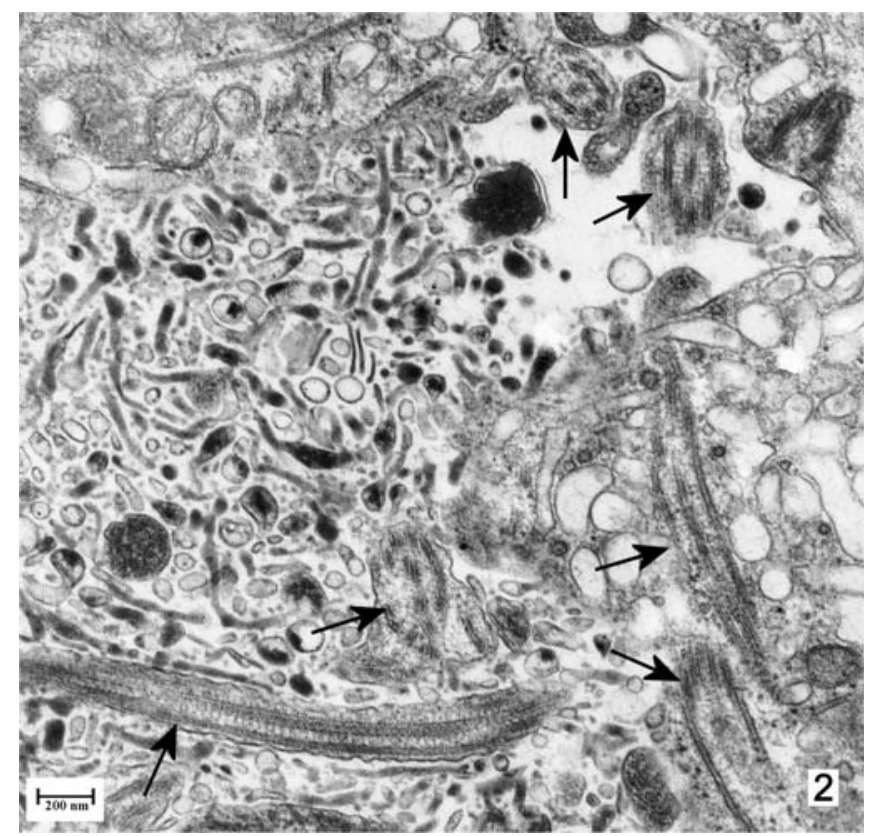

Figure 3

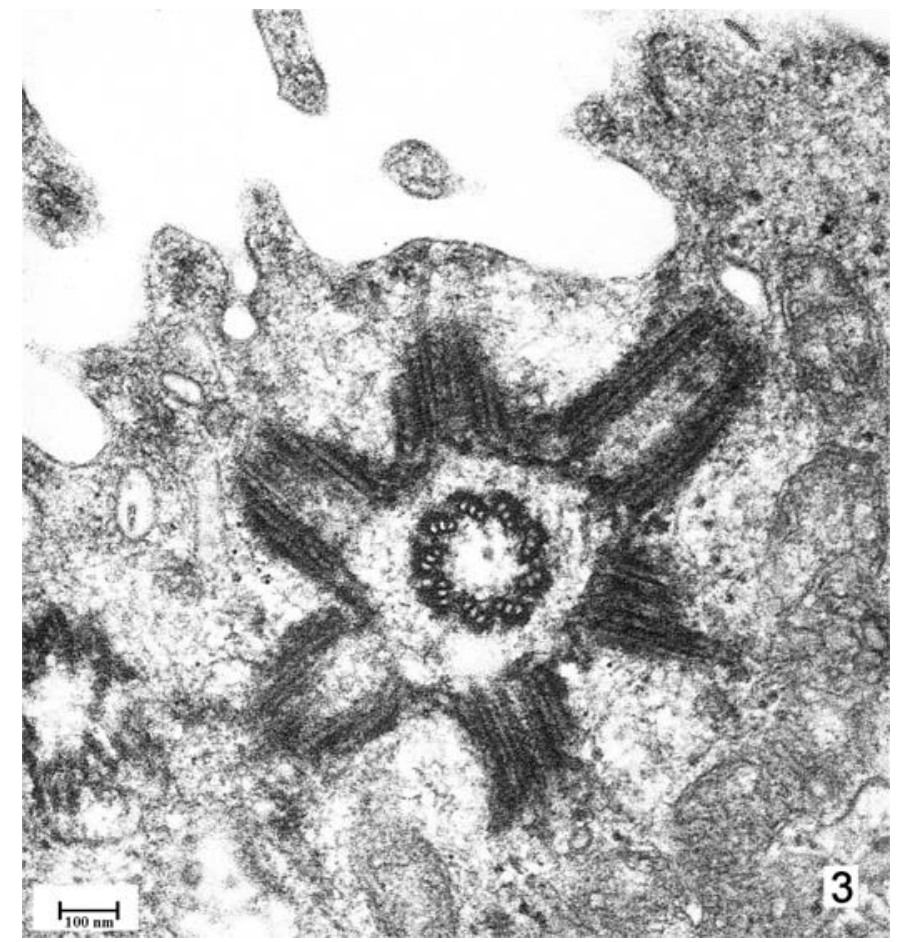

Figure 4 


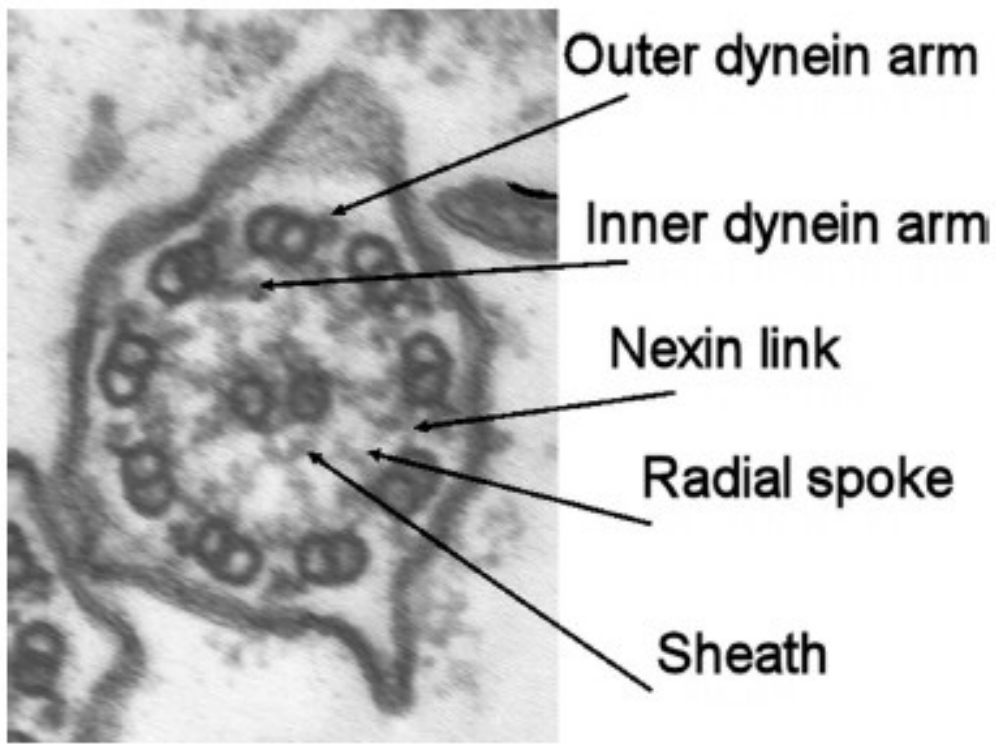

Figure 5 